\title{
TECNOLOGIAS EM ENFERMAGEM E O IMPACTO NA PRÁTICA
} ASSISTENCIAL

\author{
Camila Dannyelle Fernandes Dutra Pereira \\ Mestranda do Programa de Pós-graduação em Enfermagem, Departamento de \\ Enfermagem, Universidade Federal do Rio Grande do Norte (UFRN).

\section{camilafernandesenf@hotmail.com}

\section{Diana Paula de Souza Rêgo Pinto}

Mestranda do Programa de Pós-graduação em Enfermagem, Departamento de Enfermagem, Universidade Federal do Rio Grande do Norte (UFRN). dianarego@hotmail.com

\section{Francis Solange Vieira Tourinho}

Doutora, Professora Adjunto do Programa de Pós-Graduação em Enfermagem, Departamento de Enfermagem, Universidade Federal do Rio Grande do Norte (UFRN). Líder do Grupo de Pesquisa: Laboratório de Investigação do Cuidado, Segurança,

Tecnologias em Saúde e Enfermagem - UFRN. francistourinho@gmail.com

\section{Viviane Euzébia Pereira Santos}

Doutora, Professora Adjunto do Programa de Pós-Graduação em Enfermagem, Departamento de Enfermagem da Universidade Federal do Rio Grande do Norte (UFRN).

Vice-líder do Grupo de Pesquisa:Laboratório de Investigação do Cuidado, Segurança,

Tecnologias em Saúde e Enfermagem - UFRN. vivianeepsantos@gmail.com

\begin{abstract}
RESUMO
Objetivo: identificar as tecnologias de enfermagem e o impacto que estas causam na prática assistencial do enfermeiro. Método: Revisão integrativa da literatura, realizada em outubro de 2012, nas bases de dados LILACS, PUBMED e BDENF. Após as buscas, foi realizada leitura dos artigos selecionados, constatando-se que 34 destes atendiam aos critérios de inclusão/exclusão estabelecidos. Resultados e Discussão: Os dados evidenciaram predominância dos artigos selecionados: na Revista Brasileira de Enfermagem (REBEN), com 8 publicações (23,5\%); no ano de 2009, com 14 artigos $(41,2 \%)$; tendo como local de desenvolvimento a UTI, com 9 (26,5\%); o tipo de estudo, mais da metade foram descritivos, totalizando 17 publicações $(50,3 \%)$; dentre as tecnologias abordadas, sobressaiu as gerais na prática da enfermagem, com 16 (47\%); tratando-se da classificação destas tecnologias, em 17 artigos (50,3\%), classificou-se como dura. Já com relação ao impacto das tecnologias na assistência de enfermagem, o maior destaca foi para a categoria "Cuidado na enfermagem ao paciente" com 58,8\% dos artigos. Foi possível perceber que a prática do enfermeiro é influenciada pelas tecnologias. Conclusão: Assim, é importante que os enfermeiros busquem aperfeiçoamento da prática, sem esquecer que é o cuidado quem utiliza a tecnologia para um cuidado de enfermagem de qualidade.
\end{abstract}

PALAVRAS-CHAVE: Enfermagem. Cuidados de Enfermagem. tecnologia 


\title{
TECHNOLOGIES AND THEIR IMPACT ON NURSING CARE PRACTICE
}

\begin{abstract}
Objective: The study aimed to identify technologies nursing and the impact that these have on nursing care practice. Method: Integrative literature review, conducted in October 2012 by the databases LILACS, and PUBMED BDENF. After the search was performed reading of selected articles, noting that 34 of these met the criteria for inclusion / exclusion set. Results and Discussion: The data show a predominance of the selected articles: Brazilian Journal of Nursing (Reben), with 8 publications (23.5\%) in 2009, with 14 articles (41.2\%), having as a development site ICU with 9 (26.5\%), type of study, more than half were descriptive, totaling 17 publications (50.3\%) among the technologies discussed, excelled in the general nursing practice, $16(47 \%)$; as regards the classification of these technologies, in 17 articles $(50.3 \%)$ classified themselves as hard. Now with regard to the impact of technology in nursing care, the biggest highlight was the category for "nursing care to patients" with $58.8 \%$ of the articles. It could be observed that the practice of nurses is influenced by technology. Conclusion: Thus, it is important that nurses seek improvement of practice, without forgetting that the care is who uses technology to a nursing care quality.
\end{abstract}

KEYWORDS: Nursing. Nursing Care. Technology.

\section{TECNOLOGIAS EM ENFERMAGEM E O IMPACTO NA PRÁTICA ASSISTENCIAL}

\section{INTRODUÇÃO}

Desde os tempos mais remotos que o ser humano busca constantemente pelos avanços tecnológicos para sua sobrevivência, como com a descoberta do fogo ou dos instrumentos tecnológicos [1]. Neste sentido, a industrialização determinou avanços que promoveram a ascensão e desenvolvimento de todos os campos do conhecimento, inclusive o da saúde, com o uso da informática e de equipamentos sofisticados que possibilitaram a luta contra as doenças e a busca por melhores condições de vida e saúde [2].

Nos dias hodiernos, essa inovação tecnológica crescente fica a disposição dos profissionais e usuários. No entanto, apesar do constante e determinante uso das tecnologias, o seu conceito vem sendo utilizado de forma equívoca, pois tem sido atribuído na prática diária apenas como uma máquina ou produto. Assim, é importante destacar que as tecnologias não devem ser vistas sob um olhar reducionista associado somente a equipamentos [2].

No campo da saúde, a tecnologia não se opõe ao toque humano, mas se configura como agente e objeto deste toque. As ambiguidades das tecnologias ora produto ora significado e ora produto ora processo não a caracterizam como desumana, mas confirma seu lado objetivo e seu lado social [3]. Destarte, sua finalidade mor é tornar cada vez mais eficiente a atividade humana através da produção ou aperfeiçoamento das tecnologias, que direta e/ou indiretamente, estão a serviço do cuidado [4]. 
Estas tecnologias envolvidas no trabalho em saúde se classificam em: a) Leves: como as relações do tipo produção de vínculo, acolhimento, gestão como uma forma de governar processos de trabalho; b) Leve-duras: saberes estruturados que operam no processo de trabalho em saúde, como a clínica médica, a clínica psicanalítica e a epidemiologia; c) Duras: material concreto, como máquinas, normas, estruturas organizacionais [5].

Vale ressaltar que as três categorias estão inter-relacionadas e fazem parte da prática do cuidado, exigindo que nos estabelecimentos de saúde essas diversas tecnologias sejam constantemente contempladas para satisfazer as necessidades dos usuários [6]. No entanto, o uso destas tecnologias, apesar dos benefícios, vem gerando diversas indagações no que concerne aos possíveis impactos, riscos e as relações que se estabelecem entre os sujeitos envolvidos no processo de cuidado [1].

$\mathrm{Na}$ enfermagem, a tecnologia gera impacto significativo no processo de trabalho [7], sendo designada como a aplicação dos conhecimentos científicos de modo sistemático no auxílio para melhor atender o ser humano. É importante destacar ainda que o uso da tecnologia não deve ser entendido como paradigma de cuidado contrário ao indivíduo, e sim que pode ser fator de humanização, até mesmo nos ambientes mais tecnologicamente intensos de cuidados [3].

Desta forma, para determinar o impacto da tecnologia na assistência de enfermagem precisa-se compreender que é necessário o equilíbrio entre o uso da tecnologia e o papel desempenhado pelo enfermeiro, já que são realidades construídas socialmente [3]. Se são socialmente construídos, o enfermeiro precisa: estabelecer novas relações; buscar harmonia entre o cuidado e a tecnologia, enxergando o indivíduo além dos equipamentos [1].

O cuidado realizado pela enfermagem é um processo que envolve muitas ações e diante da complexidade para sua realização, faz-se necessária a utilização de tecnologias apropriadas [8]. Independente da tecnologia utilizada, o enfermeiro deve desempenhar um papel humanizado, lutando pelo bem-estar das pessoas, preservando seu conforto e vida, requerendo dos enfermeiros reflexão contínua acerca das suas posturas enquanto cuidadores de seres humanos [2].

A utilização das tecnologias pela enfermagem determinou repercussões no trabalho dos enfermeiros diante do redimensionamento do espaço para o cuidar, onde passaram a ter que assistir o paciente, ao mesmo que tempo que dominar os vários tipos de tecnologias [7]. Importa neste contexto, conhecer o impacto que isso determina para a prestação do cuidado integral e de qualidade.

Neste contexto tecnológico este artigo se justifica com o objetivo de identificar as tecnologias de enfermagem e o impacto que estas causam na prática assistencial do enfermeiro. Trata-se de uma temática que ainda suscita muita reflexão, apesar da tecnologia estar cada vez mais inserida na enfermagem.

\section{MÉTODOS}

Este estudo refere-se a uma revisão integrativa da literatura, uma vez que se configura como uma síntese do estado dos dados científicos relacionados a um problema de pesquisa. Trata-se de uma abordagem metodologicamente ampla referente às revisões existentes para compreender o fenômeno analisado [9], preparada a partir de etapas previamente 
estabelecidas buscando na literatura as tecnologias em enfermagem e seu impacto na assistência.

Para tal, inicialmente, elaborou-se um protocolo de revisão de literatura no qual foram definidos tema, título, questão de pesquisa, objetivo, delineamento da pesquisa, bases de dados a serem pesquisadas, descritores (via MeSH/DeCS), critérios de inclusão e exclusão e um instrumento sob forma de tabela para a coleta de dados e categorização dos resultados. A tabela em questão constava dos indicadores: título, periódico de publicação, ano de publicação, local de desenvolvimento do estudo, tipo de estudo, tecnologia abordada no artigo científico, classificação dessas tecnologias segundo Merhy(5) e o impacto dessas tecnologias na assistência de enfermagem.

A busca dos artigos foi realizada aos pares, em outubro de 2012, através das bases de dados Sistema Latino-Americano e do Caribe de Informação em Ciências da Saúde (LILACS), National Library of Medicine (PUBMED) e Banco de Dados em Enfermagem (BDENF). Foram realizados dois cruzamentos utilizando o operador booleano and: o primeiro com os descritores controlados "Enfermagem" e "tecnologia"; e o segundo com o descritor controlado "Cuidados de Enfermagem" e o descritor não controlado "inovação". A busca totalizou em 4.985 publicações, sendo que destas apenas 204 estavam disponíveis gratuitamente na íntegra nas bases de dados selecionadas.

Após isso, procedeu-se a análise crítica dos artigos, com base nos critérios de inclusão: estudos que abordassem acerca da temática proposta; artigos disponíveis na íntegra nas bases de dados selecionadas; nos idiomas português, inglês e espanhol e publicados nos últimos cinco anos (2008-2012). A opção pelos últimos 5 anos faz-se na tentativa de estudar os manuscritos mais recentes que tratam da temática em questão. Foram excluídos os artigos que abordavam as tecnologias para outros fins que não a assistencial; publicações disponíveis apenas como resumo; além dos artigos duplicados nas bases citadas e publicações do tipo dissertações, teses, editoriais, notas ao editor.

Após leitura flutuante dos títulos e resumos, excluindo-se os artigos repetidos, chegou-se a um número de 50 artigos. Em seguida, foi realizada leitura dos artigos selecionados, constatando-se que 34 destes atendiam aos critérios de inclusão/exclusão estabelecidos, sendo estes selecionados para o estudo. É importante destacar que nenhum desses artigos encontrava-se no segundo cruzamento na BDENF.

Os dados identificados nas publicações receberam tratamento descritivo através de frequências simples e percentuais em consonância com as variáveis estabelecidas previamente no instrumento de coleta de dados. Os resultados foram apresentados na forma de quadros e figuras, sendo estes discutidos a luz da literatura.

\section{RESULTADOS E DISCUSSÃO}

A síntese do processo de buscas nas bases de dados para seleção dos artigos pertinentes a este estudo, considerando os cruzamentos dos descritores (Decs/Mesh) e os critérios de inclusão/exclusão, está discriminada a seguir na Tabela 1.

\section{TABELA 1: Síntese do processo de busca dos estudos selecionados para a revisão integrativa de literatura, de acordo com bases de dados e critérios de inclusão/exclusão, 2012.}




\begin{tabular}{|c|c|c|c|c|}
\hline $\begin{array}{c}\text { Base de } \\
\text { dados }\end{array}$ & Cruzamento 1* & Cruzamento 2** & $\begin{array}{c}\text { Disponíveis na } \\
\text { íntegra }\end{array}$ & Selecionados*** \\
\hline LILACS & 93 & 12 & 42 & 13 \\
\hline PUBMED & 2698 & 1907 & 89 & 06 \\
\hline BDENF & 260 & 15 & 73 & 15 \\
\hline Total & $\mathbf{3 0 5 1}$ & $\mathbf{1 9 3 4}$ & $\mathbf{2 0 4}$ & $\mathbf{3 4}$ \\
\hline
\end{tabular}

* Cruzamento 1: Enfermagem AND tecnologia;

** Cruzamento 2: Cuidados de Enfermagem AND inovação;

*** Selecionados de acordo com critérios de inclusão/exclusão.

No que concerne aos periódicos é possível observar que a grande maioria dos artigos selecionados foram da Revista Brasileira de Enfermagem (REBEN), com 8 publicações (23,5\%); a Revista da Escola de Enfermagem da Universidade de São Paulo (REEUSP), com 3 (9\%); a Revista de Enfermagem da Universidade Estadual do Rio de Janeiro (UERJ), com 3 (9\%); a Revista Cuidado é Fundamental online, com 3 (9\%); a Revista da Escola Anna Nery, também com $3(9 \%)$ e a Online Brazilian Journal of Nursing, com 2 $(5,8 \%)$. Pode-se perceber que os periódicos mais evidenciados são de destaque para as publicações em enfermagem, contribuindo significativamente para o meio cientifico desta área.

Os demais periódicos corresponderam a uma publicação cada: Texto e Contexto Enfermagem, AQUICHAN, Heart Lung, Jornal de Pediatria, Revista Latino- Americana de Enfermagem Revista da Rede de Enfermagem do Nordeste (Fortaleza), Revista Gaúcha Enfermagem, Revista Ciência Cuidado e Saúde, Medical Informatic \& Decision Making, Quality \& Safety in Health Care, National Institutes of Health-Pediatric Emergence Care e World Journal of Sugery.

Neste estudo, optou-se pela seleção de artigos dos últimos cinco anos, período de 20082012 , buscando trabalhar com pesquisas atualizadas que versassem sobre as tecnologias em enfermagem e o impacto que o uso destas tecnologias desempenha na prática assistencial do enfermeiro. Neste contexto, pode-se destacar que dos artigos selecionados: $2(5,8 \%)$ foram de $2008 ; 14(41,2 \%)$ de $2009 ; 10(29,5)$ de $2010 ; 6(17,7 \%)$ de 2011 e 2 $(5,8 \%)$ de 2012 .

Caracterizando estes artigos com relação ao "local" de desenvolvimento do estudo: 9 nas Unidades de Terapia Intensiva $(26,5 \%) ; 7$ realizados a luz da literatura $(20,5 \%) ; 6$ nas unidades de Clinicas Médicas (17,7\%); 4 nos ambientes universitários (11,8\%); 3 nas bases de dados LILACS (Literatura Latino-americana e do Caribe em Ciências da Saúde), Medline, BDEnf (Base de Dados em Enfermagem), SciELO (Scientific Electronic Library Online), PUBMED e CINAHL (Cumulative Index to Nursing \& Allied Health Literature) (9\%); 2 em pronto-socorro $(5,8 \%) ; 2$ em outros ambientes $(5,8)$ e 1 na Atenção Básica $(2,9 \%)$.

A predominância da UTI como local de estudo corrobora com os achados da literatura ao evidenciar que na prática da enfermagem a tecnologia determina mudanças significativas, principalmente nos cuidados intensivos, já que este ambiente funciona para manutenção e recuperação da saúde através de aparelhos que exercem e/ou monitoram as funções vitais dos indivíduos momentaneamente [10].

No que se refere ao tipo de estudo, mais da metade dos artigos classificaram-se como descritivos, totalizando 17 publicações $(50,3 \%)$. Os outros estudos foram assim 
distribuídos: 7 ensaios reflexivos (20,5\%), 4 revisões de literatura $(11,8 \%), 2$ estudos experimentais $(5,8 \%), 1$ prospectivo $(2,9 \%), 1$ estudo de caso $(2,9 \%), 1$ sistêmico $(2,9 \%)$ e 1 transversal (2,9\%). Neste sentindo, é importante destacar que os estudos descritivos são caracterizados por explorar situações em que são necessárias maiores informações, pretende, portanto, descrever de maneira exata as características de determinada realidade [11].

Tratando-se das tecnologias abordadas nos estudos, estas foram categorizadas e apresentaram a distribuição, a saber no Gráfico 1.

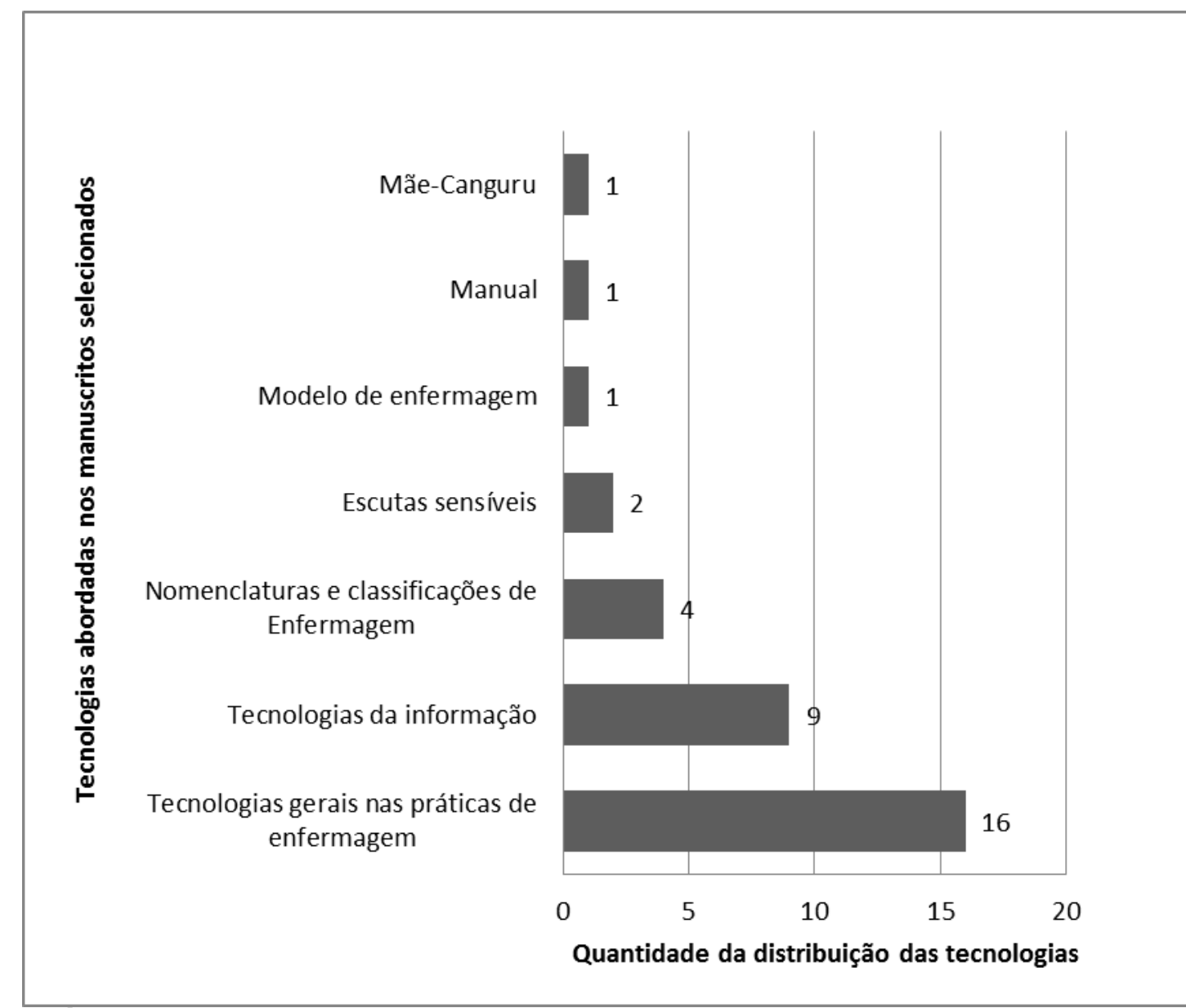

\section{GRÁFICO 1: Distribuição das tecnologias abordadas nos manuscritos selecionados para a revisão integrativa de literatura, 2012.}

Dentre do campo da enfermagem, as tecnologias podem ser classificadas com base em Merhy (2002) em leve, leve-dura ou dura. De acordo com as tecnologias abordadas nos artigos selecionados, estas foram classificadas: 3 em leve (9\%); 11 em leve-dura (32\%); 17 em dura $(50,3 \%) ; 1$ tratou de leve e levedura $(2,9 \%)$ e 2 trataram dos três tipos de tecnologias $(5,8 \%)$.

Os enfermeiros têm buscado a utilização dos recursos tecnológicos e o aproveitamento que estes podem trazer às práticas da profissão, ocasionando na aplicação prática que estes suportes podem trazer, ou seja, seus benefícios e suas vantagens. Tais recursos consistem, em sua maioria, em tecnologia duras, as quais são voltadas para os próprios profissionais [12]. 
Porém, percebe-se ainda reduzida quantidade de trabalhos que mencionam ou utilizam as tecnologias de relacionamento ou tecnologias leves. Tal fato se deva à dificuldade dos profissionais em definir o que são tais tecnologias e reconhecê-las como recursos tecnológicos [12].

Em relação aos impactos causados pelas tecnologias na enfermagem, foi realizada uma categorização e alcançou os seguintes resultados percentuais referentes a frequência dessas categorias nos manuscritos selecionados: Cuidado na enfermagem ao paciente $(58,8 \%)$, Conhecimento na prática (11,7\%), Prática direta na máquina (14,7\%), Processo de trabalho da enfermagem na prática $(38,2)$ e demais impactos $(23,5 \%)$.

No que concerne à categoria "Cuidado na enfermagem ao paciente", observou entre os autores que a tecnologia está inserida no processo de trabalho em saúde, sendo visualizada na construção do saber, nas relações entre as pessoas e no modo como ocorre o trabalho em saúde. Assim, favorece o aprimoramento do cuidado [12].

Na saúde, a tecnologia tem possibilidade de inúmeras utilizações, porém é preciso atitude reflexiva ao incorporar e perceber a sua influência no nosso "bem-viver". É necessário adequação da tecnologia à prática para o atendimento das necessidades sociais e realização do cuidado do paciente [12].

Em relação a categoria "Conhecimento na prática", a elaboração e a aplicação de um modelo de cuidado é uma forma de tecnologia, pois é uma forma de ação, um modo de fazer o cuidado. Desse modo, os modelos de cuidados mostram-se como tecnologias, que podem produzir novas tecnologias, sejam estas leves, leve-duras ou duras, que englobam um conjunto de conhecimentos para qualificar e aprimorar a práxis da Enfermagem [8].

$\mathrm{Na}$ categoria "Prática direta na máquina" observa-se que a tecnologia fornece meios e condições para avaliar procedimentos, técnicas ou instrumentos já existentes, subsidiando o desenvolvimento de novos; a utilização de instrumentos tecnológicos/máquina seria uma instância através da qual se incrementa o conhecimento de Enfermagem e se potencializa o grau de controle sobre a prática assistencial, no sentido de torná-la mais exata, mais eficaz e mais eficiente e é nessa perspectiva que está a base do incremento constante do fazer/saber enfermagem [13].

E quanto a categoria "Processo de trabalho da enfermagem na prática", observou-se que os impactos das tecnologias podem fazer com que a prática de enfermagem se torne visível no conjunto de dados, locais, nacionais e internacionais, sobre a saúde, de modo a influenciar na elaboração de políticas, tais como as de saúde e de educação. Por exemplos, as tecnologias de informação, essenciais para a tomada de decisões eficazes e para uma prática de qualidade, favorecendo o processo de trabalho de forma que os conhecimentos profissionais adquiridos possam ajudar a conhecer e a compreender melhor os assuntos relacionados com a atenção à saúde [14].

Por fim, a categoria "Demais impactos" incluiu os impactos a saber: Melhoria da saúde ocupacional; Enfermagem home care; Benefício a formação centrada na cirurgia baseada em evidências; Reorienta o fluxo de atendimento no pronto-socorro e Confere às ações de enfermagem uma amplitude de sensibilidade, ética, estética e solidariedade humana. 
Isto vai de encontro com a literatura, em que o conhecimento serve de base e suporte para os profissionais de enfermagem que cuidam, pois o teórico (conhecimento) e o técnico (conduta) se aliam à cientificidade do fazer profissional, gerando o cuidado. Salienta-se ainda que, com o conhecimento necessário e a utilização das tecnologias com ações bem planejadas, é possível prevenir os erros e danos causados ao paciente, melhorando a qualidade de assistência prestada no cuidado com a saúde [15].

\section{CONSIDERAÇÕES FINAIS}

A assistência de enfermagem, nos dias hodiernos, sofre grandes impactos das tecnologias em todas as áreas de atendimento. Diante dos dados encontrados neste estudo, foi possível perceber que a prática do enfermeiro é primordialmente influenciada pelas tecnologias duras, seguida das leve-duras, estando as leves ainda sendo pouco valorizadas e implementadas pelos enfermeiros para o cuidado.

Foi possível identificar ainda que, dentre as influências que a prática de enfermagem sofre mediante os avanços das tecnologias e seu uso, os maiores impactos se dão na categoria "Cuidado na enfermagem ao paciente", a qual aponta que as tecnologias atuam de modo a fortalecer e qualificar o cuidado, além de permitir a tomada de decisão mediante o raciocínio clinico, levando em consideração a individualização do indivíduo.

Assim, é importante que os enfermeiros se adaptem a esse contexto de avanços, buscando qualificação e aperfeiçoamento da prática, sem esquecer que é o cuidado quem utiliza a tecnologia em direção a um cuidado de enfermagem mais eficaz e seguro. Neste interim, os profissionais devem estar em constante processo de reflexão.

\section{REFERENCIAS}

1. Schwonke CRGB, Lunardi Filho WD, Lunardi VL, Santos SSC, Barlem EL. Perspectivas filosóficas do uso da tecnologia no cuidado de enfermagem em terapia intensiva. Rev Bras Enferm, Brasília. [on-line]. 2011 jan-fev. [citado em 03 nov 2012]; 64(1): 189-92. Disponível em: http://www.scielo.br/pdf/reben/v64n1/v64n1a28.pdf

2. Barra DCC, Nascimento ERP, Martins JJ, Albuquerque GL, Erdmann AL. Evolução histórica e impacto da tecnologia na área da saúde e da enfermagem. Revista Eletrônica de Enferm. [on-line]. 2006 [citado em 03 nov 2012]; 8(3): 422 - 430. Disponível em http://www.fen.ufg.br/revista/revista8 3/v8n3a13.htm

3. Martins CR, Dal Sasso GTM. Tecnologia: definições e reflexões para a prática em saúde e enfermagem. Texto Contexto Enferm, Florianópolis. [on-line]. 2008 jan-mar. [citado em 03 nov 2012]; 17(1): 11-2. Disponível em: http://www.scielo.br/pdf/tce/v17n1/01.pdf

4. Koerich MS, Backes DS, Scortegagna HM, Wall ML, Veronese AM, Zeferino MT, et al. Tecnologias de cuidado em saúde e enfermagem e suas Perspectivas filosóficas. Texto Contexto Enferm, Florianópolis. [on-line]. 2006[citado em 04 nov 2012]; 15 (Esp): 178-85. Disponível em: http://www.scielo.br/pdf/tce/v15nspe/v15nspea22.pdf

5. Merhy EE. Saúde: a cartografia do trabalho vivo. São Paulo: HUCITEC; 2002.

6. Rossi FR, Lima MADS. Fundamentos para processos gerenciais na prática do cuidado. Rev Esc Enferm USP. [on-line]. 2005 [citado em 04 nov 2012]; 39(4):460-8. Disponível em: http://www.ee.usp.br/reeusp/upload/pdf/68.pdf 
7. Silva RC, Ferreira MA. A tecnologia em saúde: uma perspectiva psicossociológica aplicada ao cuidado de enfermagem. Esc Anna Nery Rev Enferm. [on-line]. 2009 janmar. [citado em 04 nov 2012]; 13 (1): 169-173. Disponível em: http://www.scielo.br/pdf/ean/v13n1/v13n1a23.pdf

8. Rocha PK, Prado ML, Wal ML, Carraro TE. Cuidado e tecnologia: aproximações através do Modelo de Cuidado. Rer Bras Enferm. [on-line]. 2008 [citado em 03 nov 2012]; 61(1):113-6. Disponível em: http://www.scielo.br/pdf/reben/v61n1/18.pdf

9. Souza MT, Silva MD, Carvalho R. Revisão integrativa: o que é e como fazer. Einstein. [on-line]. 2010 [citado em 03 nov 2012]; 8:102-6. Disponível em: http://apps.einstein.br/revista/arquivos/PDF/1134-Einsteinv8n1_p102-106_port.pdf

10. Silva RC, Ferreira MA. Tecnologia na terapia intensiva e suas influências nas ações do enfermeiro. Rev Esc Enferm USP. [on-line]. 2011 [citado em 04 nov 2012]; 45(6):1403-1. Disponível em: http://www.scielo.br/pdf/reeusp/v45n6/v45n6a18.pdf

11. Leopardi MT. Metodologia da pesquisa em saúde. 2a . ed. Florianópolis: UFSC/PósGraduação em Enfermagem; 2002.

12. Lopes EM, Pinheiro AKB, Pinheiro PNC, Vieira NFC. Tecnologia e práticas de enfermagem - um estudo bibliográfico. Online Brazilian Journal of Nursing. [on-line]. 2009 [citado em 05 nov 2012]; 8(1). Disponível em: http://www.objnursing.uff.br/index.php/nursing/article/view/j.1676$\underline{4285.2009 .1883 / 446}$

13. Vargas MAO, Ramos FRS. Tecnobiomedicina: implicações naquilo e daquilo que a enfermagem faz em terapia intensiva. Texto Contexto Enferm, Florianópolis. [online]. 2008 jan-mar. [citado em 05 nov 2012]; 17(1): 168-76. Disponível em: http://www.scielo.br/pdf/tce/v17n1/19.pdf

14. Nóbrega MML, Garcia TR. Classificação Internacional para a Prática de Enfermagem: instrumental tecnológico para a prática profissional. Rev Bras Enferm, Brasília. [online]. 2009 set-out. [citado em 05 nov 2012]; 62(5): 758-61. Disponível em: http://www.scielo.br/pdf/reben/v62n5/19.pdf

15. Silva MA, Torres GV, Melo GSM, Costa IKF, Tiburcio MP, Farias TYA. Conhecimento acerca do processo transfusional da equipe de enfermagem da uti de um hospital universitário. Cienc Cuid Saude. [on-line]. 2009 out-dez. [citado em 05 nov 2012]; 8(4):571-578. Disponível em: http://periodicos.uem.br/ojs/index.php/CiencCuidSaude/article/view/9676/5389 